\title{
LIMITER LOCK SYSTEMS AT TEXTOR: FLEXIBLE TOOLS FOR PLASMA-WALL INVESTIGATION
}

\author{
B. SCHWEER,* S. BREZINSEK, H. G. ESSER, A. HUBER, PH. MERTENS, S. MUSSO, \\ V. PHILIPPS, A. POSPIESZCZYK, U. SAMM, G. SERGIENKO, and P. WIENHOLD \\ Institut für Plasmaphysik, Forschungszentrum Jülich GmbH, EURATOM Association \\ Trilateral Euregio Cluster, D-52425 Jülich, Germany
}

Received June 11, 2004

Accepted for Publication August 23, 2004

Limiter lock systems on the top and the bottom of the TEXTOR vessel are essential elements for experimental investigations of plasma-wall interaction in a tokamak. The lock systems are designed as user facilities that allow the insertion of wall elements (limiter) and tools for diagnostic (electrical probes, gas injection) without breaking the TEXTOR vacuum. The specially designed holder on top of the central carrier and a powerful vacuum pump system permit the exchange of components within $\sim 1$ h. Up to ten electrical signals, four thermocouples, and a gas supply can be connected at the holder interface. Between discharges, the inserted component can be positioned radially and turned with respect to the toroidal magnetic field. Additionally, the central carrier is electrically isolated to apply bias voltages and currents up to $1 \mathrm{kV}$ and $1 \mathrm{kA}$, respectively.
An important feature of the lock system is the good access for optical spectroscopic observation of the inserted components in the vicinity of the edge plasma. The whole spectrum from ultraviolet to infrared is covered by spectrometers and filters combined with cameras. Toroidally and poloidally resolved measurements are obtained from the view on top of the probes while the tangential poloidal view delivers radially and toroidally resolved information.

A programmable logic controller (Simatic S5) that is operated inside the TEXTOR bunker and from remote locations outside the concrete wall drives all possible features of the lock system.

KEYWORDS: limiter lock, plasma edge spectroscopy, plasmasurface interaction

\section{INTRODUCTION}

At TEXTOR plasma-wall interaction is one of the main aims of investigation that was considered during the design of the vessel. The vessel elements are equipped with large ports, and especially the tangential ports in all poloidal cross sections give access to the edge region of the plasma. The plasma radius of TEXTOR can be varied between 410 and $480 \mathrm{~mm}$ by positioning either poloidal limiters at the top and the bottom of the vessel or the toroidal belt limiter ${ }^{1}$ ALT II. Most of the discharges were performed with a plasma radius of $460 \mathrm{~mm}$. This is, on one hand, a compromise to get a large plasma radius and, on the other hand, to establish a large distance to components installed inside the liner (which is at a radius of $546 \mathrm{~mm}$ ) and the vessel. ${ }^{2}$

\footnotetext{
*E-mail: B.Schweer@fz-juelich.de
}

The radial distance from the last closed flux surface (LCFS) defined by the limiters (mainly ALT II at $460 \mathrm{~mm}$ ) through the scrape-off layer (SOL) to the wall components (plasma boundary) is $\sim 100 \mathrm{~mm}$ over the entire poloidal section, and this is very advantageous for studying plasma-surface interaction (PSI). Plasma-wall interaction effects require especially high spatial resolution directly at the material surface.

The flexible positioning of material elements and diagnostic probes at any position inside the SOL does offer a wide field for investigation of PSI. Therefore, at TEXTOR two so-called limiter locks have been developed and mounted at different toroidal sections on the bottom (section 10/11) and the top (section 15/16) of the vessel.

A main feature of the limiter lock concept is the good access for spectroscopic and optical observation in the horizontal as well as in the vertical direction, allowing the measurement of plasma parameters in close vicinity 
to the probe head. Combined with these diagnostic tools, the effects of poloidal and toroidal asymmetries can be investigated and are a valuable input for modeling.

The lock systems are designed to fulfill the following requirements:

1. short exchange times for samples and probes combined with high reliability

2. vacuum conditioning by internal heating of the samples

3. active resistive heating to very high temperatures $(1500 \mathrm{~K})$

4. gas injection through samples

5. rotation of probes between discharges

6. interfaces for thermocouples, electrical power, and signal lines

7. remote operation of positioning and gas injection

8. selective reference potential of probe carrier

9. vertical and horizontal access for optical and spectroscopic observation of probes.

The features of both lock systems are similar in principle. Therefore, only a description of the bottom lock system (limiter lock 1) is given in this paper that is organized as follows:

1. description of the lock properties

2. operational features

3. standard test limiter head

4. description of the observation system.

\section{VACUUM LOCK COMPONENTS}

The setup for limiter lock 1 at the bottom of the TEXTOR vessel is shown in Fig. 1. The linear motion drive with edge-welded bellows, the central carrier with target exchange interface, the probe exchange chamber, the intermediate vacuum chamber with carrier clamps for stabilization, the vacuum pump system, and the SPS (Speicher programmierbare Steuerung) control system are the main parts of the lock system. ${ }^{3}$

\section{Linear Motion Drive}

The linear motion drive consists of two CF100 flanges. One moves along two parallel rods and is linked to a motor-driven ball bearing spindle. The flanges are interconnected with edge-welded bellows allowing a motion over a full length of $1750 \mathrm{~mm}$ under ultrahigh vacuum conditions.

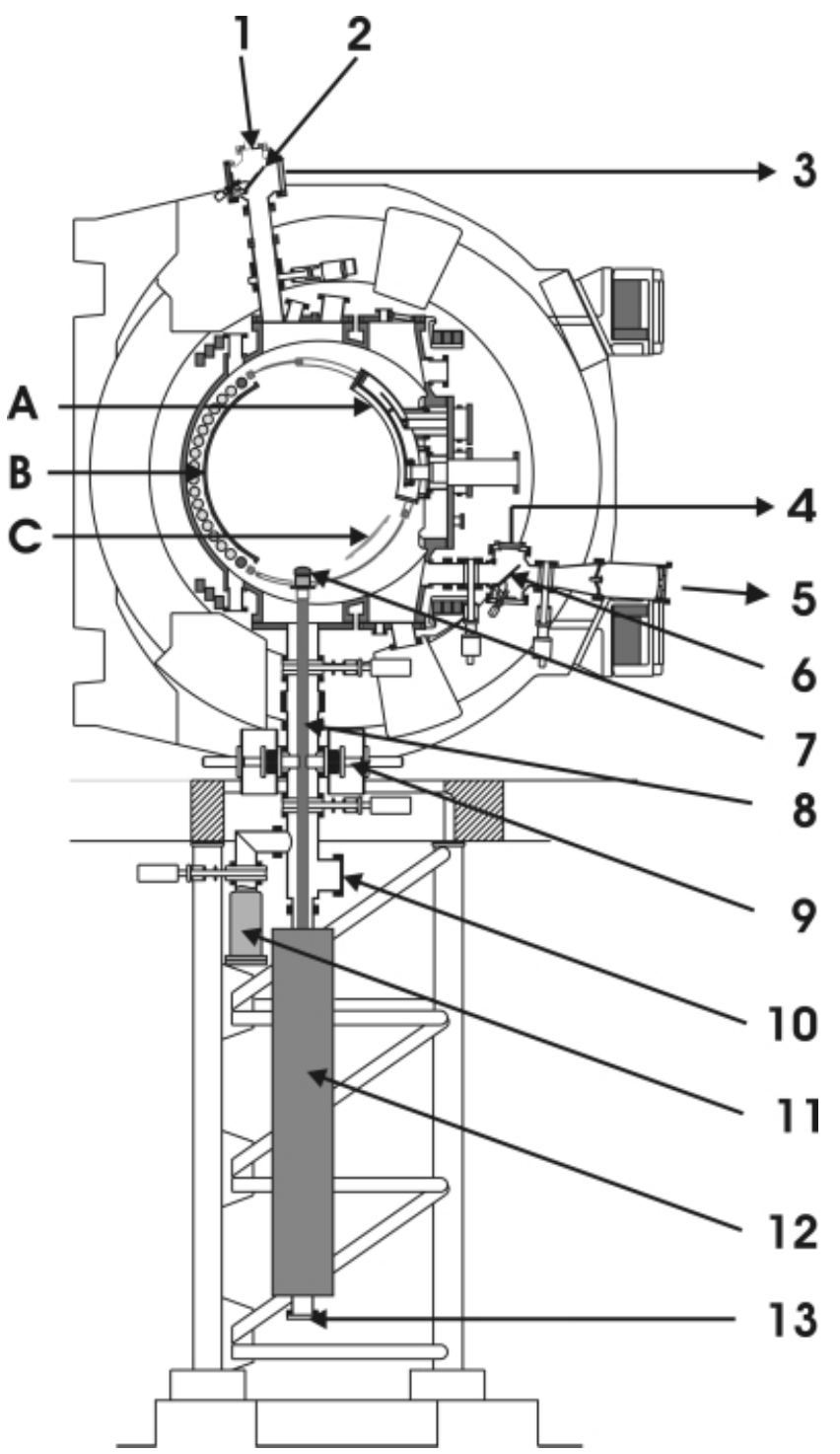

Fig. 1. Poloidal cut of the bottom limiter lock system with observation ports at TEXTOR: 1. laser entrance port (LIF); 2. pivot mirror; 3. vertical observation (ultraviolet to infrared); 4 . horizontal observation (ultraviolet to infrared); 5 . VUV observation; 6 . pivot mirror; 7. exchangeable target or probe (test limiter); 8. central carrier; 9. piston for stabilization; 10. loading port; 11. cryopump; 12 . linear motion unit; 13 . feedthroughs to air for electrical signals and power, gas, and target rotation; A. ion cyclotron resonance heating antenna; B. dynamic ergodic divertor tiles; C. ALT II toroidal limiter.

\section{Central Carrier}

Attached at the movable CF100 flange, a central carrier tube of $2000 \mathrm{~mm}$ length is inserted into the vacuum system. It consists of three coaxial tubes. At one end (far from the plasma), the inner and the middle tubes are 
welded on a CF100 cap that, isolated by a Vespel ring, is mounted on the movable flange. At the other side (toward the plasma), each of these tubes is connected via bellows to a movable piston at the probe exchange interface. The enclosed volume can be pumped by a separate rotary pump (V8 open and V9 closed) or pressurized by air or nitrogen (V8 closed and V9 open) to release or fix, respectively, the probe by the piston (Fig. 2). The inner tube is used mainly for gas injection supply but can also carry water tubes for target cooling. The $82-\mathrm{mm}$ diam outer tube is fixed by screws to the CF100 cap without vacuum sealing. It stabilizes the carrier, screens

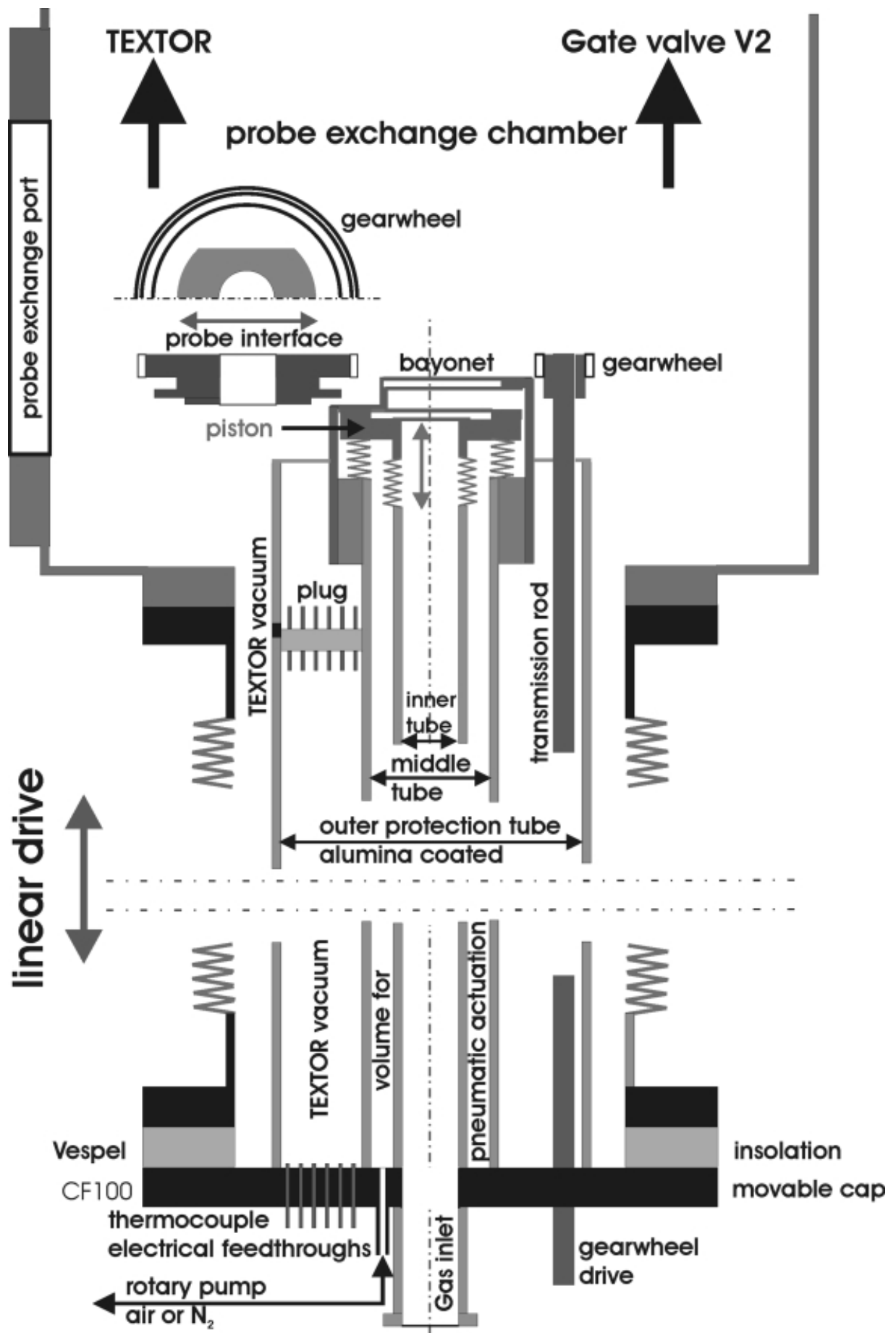

Fig. 2. Flow diagram of the vacuum system at the bottom limiter lock. 
the cabling mounted on the intermediate tube, and holds the support for the bayonet holder and the piston. The electrical interface accessible at the exchange chamber is provided with plug-in connectors for the connection of two "type C" thermocouples for high-temperature measurements up to $2320^{\circ} \mathrm{C}$ and two "type $\mathrm{K}$ " thermocouples for measurements of medium temperatures up to $1250^{\circ} \mathrm{C}$. Additional plug-in connectors for electrical circuits consist of four wires and ten wires with maximum currents of $15 \mathrm{~A}$ and $1 \mathrm{~A}$, respectively. Feedthroughs mounted at the CF100 cap provide the electrical connection from vacuum to air.

All electrical cables on the "air" side are tested to an insulation voltage up to $2 \mathrm{kV}$. A power supply for probe heating and all amplifiers for temperature measurements are permanently coupled by isolated transformers.

A rotary motion is transferred via a feedthrough and a rod to the gear transmission (4:1) at the interface support. All probes or targets must be mounted on a probe interface block (see Fig. 2) that fits into the bayonet holder and gearbox.

The central carrier is electrically isolated with respect to the supporting linear drive system. High currents up to $1000 \mathrm{~A}$ at a maximum voltage of $1 \mathrm{kV}$ allow biasing experiments. For safety reasons, the vacuum system is also isolated to TEXTOR; any current, which may occur by arcs inside the lock system, is detected and forces a switch-off of the biasing voltage.

\section{Target Exchange Chamber}

The driving unit is connected to the exchange chamber that is basically a $150-\mathrm{mm}$-diam tube equipped with additional ports mainly for loading and pumping.

An important feature of the locks is a fast exchange of targets. For this purpose, powerful pump systems are applied. The principle setup of the vacuum system is shown in Fig. 3.

The limiter lock at the bottom of the TEXTOR vessel is connected to a cryogenic pump with $1.5 \mathrm{~m}^{3} / \mathrm{s}$ pumping speed, separated by a gate valve (V4). The basic pressure to open this valve and to apply the full pumping speed of the cold cryopump is provided via a gate valve (V6) by a turbomolecular pump system. Exchange times of $\sim 1 \mathrm{~h}$ are possible if the system is vented with $\mathrm{N}_{2}$ (valve V7) but might be prolonged by outgassing of the probes.

\section{Intermediate Vacuum Chamber}

In spite of the longer linear motion required by this arrangement, each limiter lock is supplied with an intermediate vacuum chamber between TEXTOR and the target exchange chamber. Even in case of leakage of the main gate valve (V1) to TEXTOR, a target exchange is still possible. During this operation the second gate valve (V2) is closed. The gate valve (V3) to the turbomolecular pump is opened, and the volume is evacuated indepen-

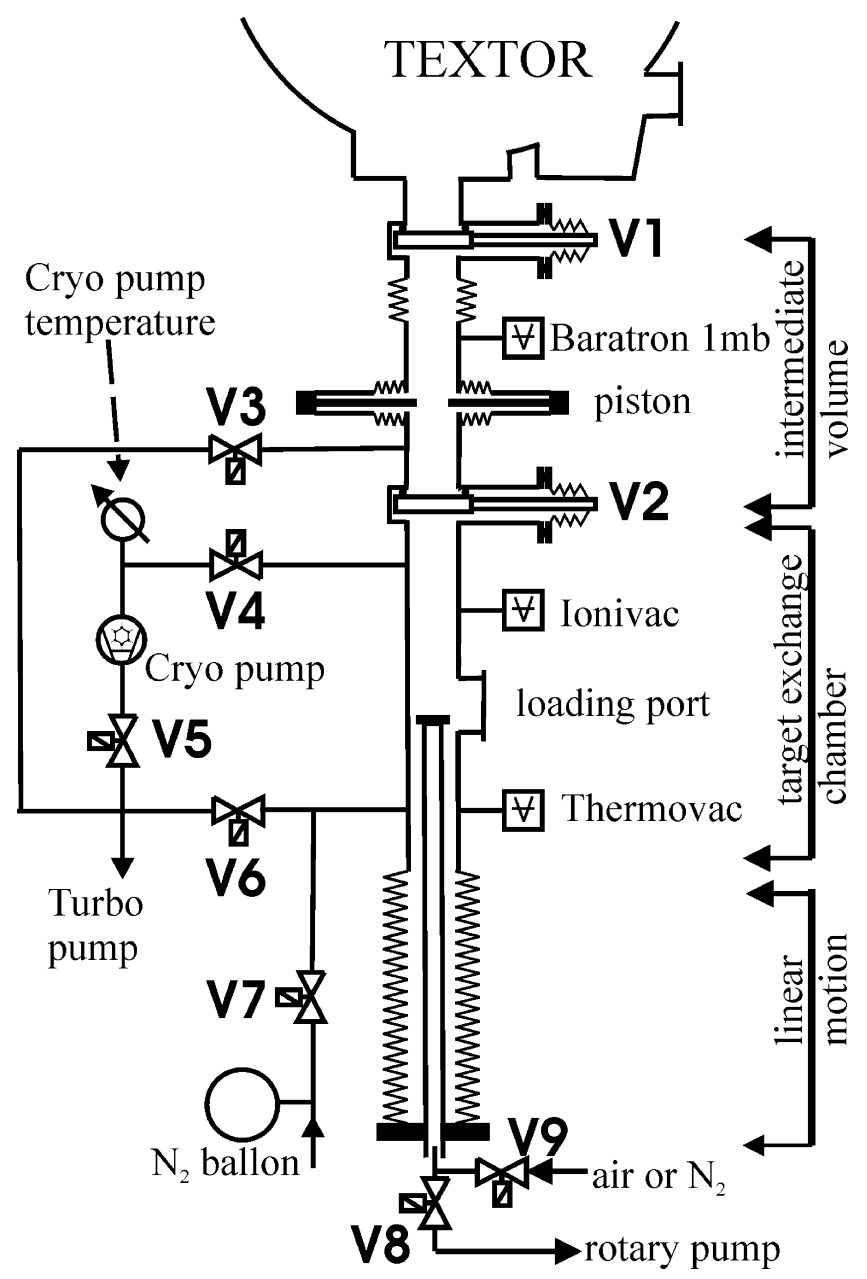

Fig. 3. Principal arrangement of the interface for target exchange.

dently to avoid a pressure rise inside the TEXTOR vessel. Therefore, the reliability of the lock is increased significantly. For the actual measurements, the central carrier transports the limiter or probe through the 150$\mathrm{mm}$-diam tube to the desired position at the main plasma chamber. Inside the intermediate volume, two electrically isolated pistons clamp the tube pneumatically.

\section{Target Interface}

All targets must be mounted onto a standard exchangeable interface block that fits into both limiter locks. Figure 2 shows the principal layout and function of the interface.

The outer dimensions of the targets are limited by the 150-mm inner diameter of the transfer tube to TEXTOR and by the size of the loading ports that are $150 \mathrm{~mm}$ at the bottom and $200 \mathrm{~mm}$ at the top lock system. For safety reasons, the distance of the probe to the wall inside the transfer tube should not go down below $10 \mathrm{~mm}$. 
As described before, the piston at the interface is retracted if the volume between the inner and middle tube is evacuated. The small width of the interface is moved into the bayonet support on top of the central carrier and secured against independent removal by a 90-deg turn. The piston will fix the target if the exchange chamber is evacuated and the volume behind the piston is vented to normal pressure. For special events the pressure can be increased up to 10 bars to avoid rotation of the target by electromagnetic forces.

The movable interface is equipped with a gear wheel that forms a gearbox with a smaller wheel at the exchange interface. This allows adjustment of the target orientation with respect to the magnetic field in small angles of rotation between discharges. For this operation the volume behind the piston is pumped to reduce its force on the interface. Also, full rotation of $\pm 180 \mathrm{deg}$ is possible if the probe is mounted on a ball bearingsupported disc that forms a gearbox with 4 to 1 transmission.

\section{CONTROL SYSTEM}

A programmable logic controller (SPS) controls the limiter lock and executes the following scenarios:

\section{Start-Up}

This program automatically produces vacuum conditions inside the lock system as needed for TEXTOR operation. It opens valves V3, V5, and V6 and starts the turbopump system. The pressure inside the exchange chamber is measured by a thermoconductive gauge within the range of $10^{3}$ to $10^{-3} \mathrm{mb}$ (Thermovac) and by an ionization gauge for values below $10^{-3} \mathrm{mb}$ (Ionivac). At $5 \times 10^{-3} \mathrm{mb}$, the compressor of the cryopump is switched on. If this pump has reached a temperature of $20 \mathrm{~K}$, the valves to the turbomolecular pump are closed and the gate valves $\mathrm{V} 2$ to the intermediate volume and V4 to the cryopump are opened.

\section{TEXTOR Operation}

From start-up the intermediate gate valve V2 is open, and the pressure measured by a baratron is below $1 \mathrm{mb}$. The central control then allows the opening of the gate valve V1 to TEXTOR by the operator and a movement to a preselected radial position. A rope sensor measures the position independently with an absolute accuracy of $\pm 1 \mathrm{~mm}$ over the whole range. This mode of operation is accessible from several remote locations inside and outside the TEXTOR bunker, especially at the diagnostic room.

\section{Target Exchange}

If this program is selected, the central carrier moves back to the initial position. The gate valves to TEXTOR
(V1), the intermediate volume (V2), and cryopump (V4) are closed. The turbopump is switched on, and a valve (V3) to the intermediate volume is opened. The exchange chamber is vented via valve V7 with nitrogen to normal pressure. A rotary pump evacuates the volume behind the piston that will be retracted if valve V8 is opened and V9 closed (Fig. 3). Through the opened exchange port, the limiter or probe including all electrical connections can then be removed or inserted. If the port is closed, the program is started again manually and the exchange chamber is evacuated down to $5 \times 10^{-3} \mathrm{mb}$ by the turbomolecular pump. At this threshold, the valve V6 is closed and, simultaneously, the gate valve V4 to the cryopump is opened. The pressure normally drops to $\sim 10^{-6} \mathrm{mb}$ within seconds. Over a panel the operator only has to encode the height of the probe (offset) to adjust the correct radial position for positioning inside TEXTOR. Usually the probes are heated up to $400^{\circ} \mathrm{C}$ to avoid outgassing by plasma exposure.

Two additional features have to be described.

\section{Gas Injection System}

The gas injection system consists of a $2-\ell$ reservoir that can be evacuated by a rotary pump. The gas flow from a gas bottle is regulated by a preadjusted needle valve. The filling pressure inside the reservoir is adjustable through the Simatic S5 control and measured by a $10-$ or $100-\mathrm{mb}$ Baratron gauge. For injection a valve at the lock system is opened by a gate signal from the TEXTOR timing. If the reservoir pressure is falling below the threshold, a valve will be opened by the control system after a 15-s delay to avoid refilling during the discharge. A CAMAC data logger also records the pressure drop at the reservoir.

\section{Support for Test Limiter}

As described earlier, the targets designed by the user must be mounted on a standard movable interface (Fig. 2). In case of experiments with limiters, a special design has been made that is shown in Fig. 4. The limiter, made of different materials, is a half sphere with $6.5 \mathrm{~cm}$ radius. It is mounted on a stainless steel plate wherein a 2-m-long Thermocoax wire with 2-mm outer diameter is embedded, which can be electrically heated with maximum power of $1 \mathrm{~kW}$ corresponding to $\sim 10 \mathrm{~W} / \mathrm{cm}^{2}$. The maximum temperature of the plate is limited to $1000^{\circ} \mathrm{C}$. For carbon limiter tiles, surface temperatures of $700^{\circ} \mathrm{C}$ have been obtained without plasma heating. Higher temperatures up to $1200^{\circ} \mathrm{C}$ are achieved with an electrical heater plate consisting of pyrolytic carbon layers embedded in boron nitride (Boralectric). Both heating plates are isolated toward the lock interface by a 10-mm-thick, highly oriented pyroelectric graphite (HOPG) plate that has an extremely low heat conductance across the layers. Two thermocouples (type $\mathrm{K}$ or type $\mathrm{C}$ ) are located $5 \mathrm{~mm}$ below 


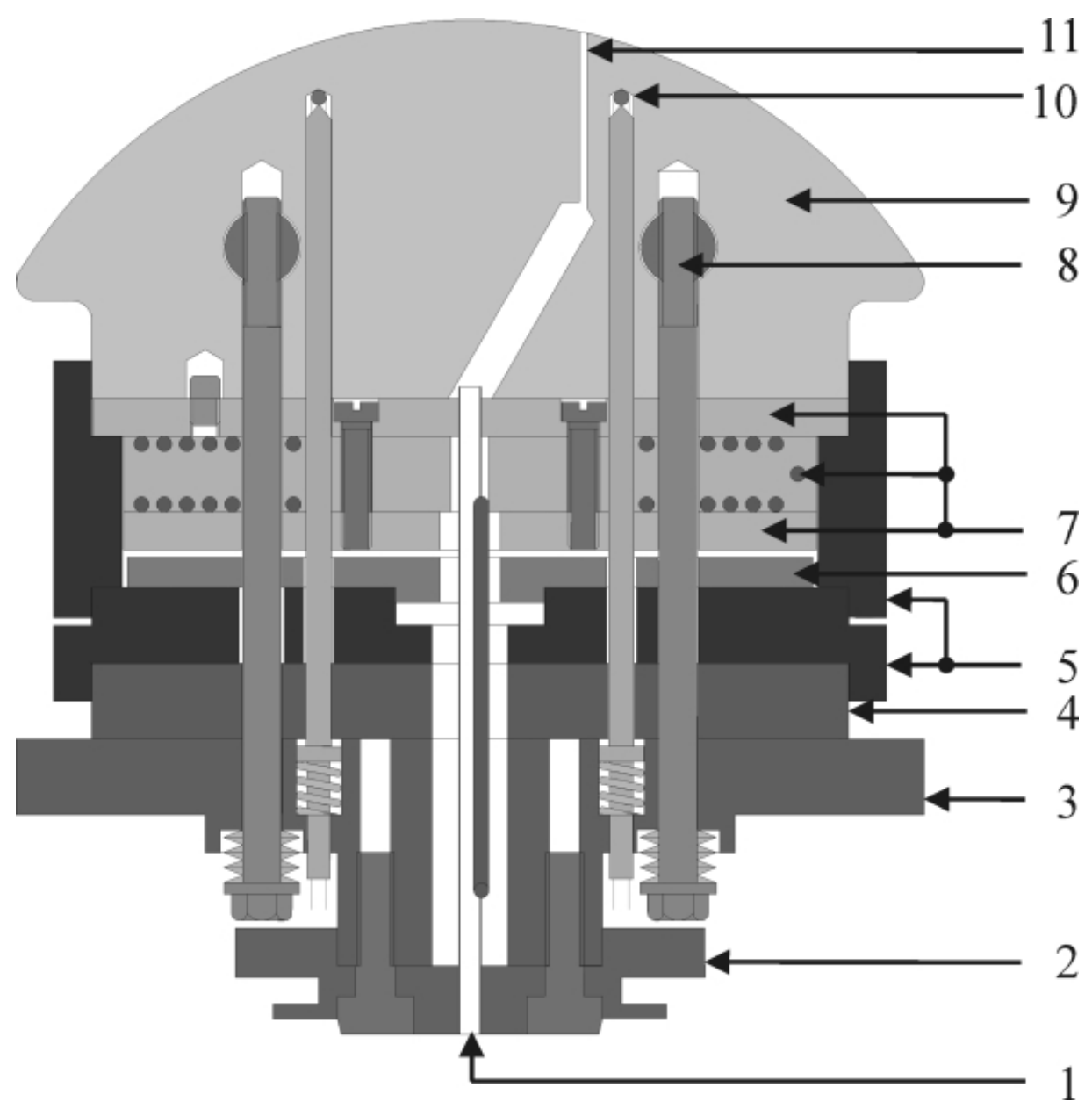

Fig. 4. Toroidal cut of the exchangeable test limiter unit: 1. gas inlet, 2. geared interface, 3. base plate, 4. HOPG plate, 5. carbon housing, 6. stainless steel reflection plate, 7. heating plate (Thermocoax), 8. assembling screws, 9. limiter head, 10. thermocouples, 11. gas injection hole.

the surface and $40 \mathrm{~mm}$ apart in toroidal direction, symmetrically on both sides of the limiter tip. Optionally for gas injection experiments, some limiter heads are equipped with a channel to the central tube of the carrier.

\section{OBSERVATION AND DIAGNOSTICS}

Optical access in vertical and poloidal, horizontal direction to the samples positioned at the plasma edge allows intensive simultaneous investigation of the plasmasurface interaction. The standard diagnostic available at the bottom limiter lock is described in the following chapters.

\section{Horizontal Observation}

A 100-mm-diam gate valve can close the horizontal port centered $500 \mathrm{~mm}$ from the tokamak midplane. At- tached to that, electrically isolated by a Vespel ring, is a vacuum chamber with a Cassegranian telescope equipped with a solar blind multiplier for vacuum ultraviolet (VUV) measurements that is mainly used for laser-induced fluorescence (LIF). The whole system can be tilted by $\sim 6 \mathrm{deg}$ out of the horizontal plane for radial scans in the range from 550 to $400 \mathrm{~mm}$. Toroidal positions are adjusted by tilting the Cassegrain main mirror.

For measurements at wavelengths above $200 \mathrm{~nm}$, the plasma light is deflected through a quartz window by a pivoted mirror inside the vacuum chamber that can be positioned remotely. Outside the vacuum, mirrors and quartz lenses guide the light to several spectroscopic observation systems. ${ }^{4,5}$

These are inside the bunker:

1. a two-dimensional (2-D) observation by an intensified charge-coupled device (CCD) camera (Proxitronic TV camera, 40-ms time resolution) with interference filters and neutral filters mounted on 
TABLE I

Parameter of the ARC Pro500 Spectrometer

\begin{tabular}{|c|c|c|c|}
\hline $\begin{array}{c}\text { Grating } \\
\text { Number }\end{array}$ & $\begin{array}{c}\text { Groove } \\
\text { Density }\end{array}$ & $\begin{array}{c}\text { Dispersion } \\
1 \text { st Order }\end{array}$ & $\begin{array}{c}\text { Resolution } \\
50 \mu \mathrm{m} \text { Slit }\end{array}$ \\
\hline 1 & $600 / \mathrm{mm}$ & $1.6 \mathrm{~nm} / \mathrm{mm}$ & $0.08 \mathrm{~nm}$ \\
2 & $1200 / \mathrm{mm}$ & $0.8 \mathrm{~nm} / \mathrm{mm}$ & $0.04 \mathrm{~nm}$ \\
3 & $2400 / \mathrm{mm}$ & $0.4 \mathrm{~nm} / \mathrm{mm}$ & $0.02 \mathrm{~nm}$ \\
\hline
\end{tabular}

three wheels in a light-tight housing that are remotely exchangeable.

2. an imaging, 500-mm focal length spectrometer (ARC Spectra Pro500) with three remotely selectable gratings with intensified CCD camera (Proxitronic TV camera). The radial resolution is $\Delta r=0.5 \mathrm{~mm}$, and spectral properties are given in Table I.

3. an imaging, 270-mm focal length spectrometer with similar specifications but with a different set of gratings listed in Table II.

Additionally, light is coupled into an array of fibers that are connected with spectrometers outside the concrete shielding as follows:

1. a very high resolution, 1-m focal length, Echelle spectrometer equipped with an intensified 2-D CCD camera (Dalsa $1 \mathrm{M}$ ) with $30-\mathrm{Hz}$ maximum repetition rate. ${ }^{6}$ The Echelle grating with a groove density of $79 / \mathrm{mm}$ and a blaze angle of $70 \mathrm{deg}$ is operated in a very high dispersion order $m$. The dispersion at $m=46$ is $0.1 \mathrm{~nm} / \mathrm{mm}$.

2. a high-resolution, $0.8-\mathrm{m}$ focal length spectrometer with a 2-D optical multichannel system mainly used for measurements of molecular spectra. ${ }^{7}$ The grating with a groove density of $1200 / \mathrm{mm}$ and a blaze angle of $46 \mathrm{deg}$ is operated in $2 \mathrm{nd}$ order $(m=2)$. The dispersion is $0.34 \mathrm{~nm} / \mathrm{mm}$.

TABLE II

Parameter of the ARC Pro270 Spectrometer

\begin{tabular}{|c|c|c|c|}
\hline $\begin{array}{c}\text { Grating } \\
\text { Number }\end{array}$ & $\begin{array}{c}\text { Groove } \\
\text { Density }\end{array}$ & $\begin{array}{c}\text { Dispersion } \\
\text { 1st Order }\end{array}$ & $\begin{array}{c}\text { Resolution } \\
50 \mu \mathrm{m} \text { Slit }\end{array}$ \\
\hline 1 & $300 / \mathrm{mm}$ & $5.93 \mathrm{~nm} / \mathrm{mm}$ & $0.296 \mathrm{~nm}$ \\
2 & $600 / \mathrm{mm}$ & $2.96 \mathrm{~nm} / \mathrm{mm}$ & $0.148 \mathrm{~nm}$ \\
3 & $1200 / \mathrm{mm}$ & $1.48 \mathrm{~nm} / \mathrm{mm}$ & $0.074 \mathrm{~nm}$ \\
\hline
\end{tabular}

\section{Vertical Observation System}

The vertical observation is performed through a $100-\mathrm{mm}$ port with a gate valve. An electrically isolated vacuum chamber equipped with a pivoted mirror is attached. Usually the mirror is turned into a position where the light is deflected through a quartz window.

Inside the bunker, the light radiation is analyzed by

1. a 2-D observation with a CCD camera (Hitachi TV camera) with interference filters. If the filter is replaced by a long-pass $(850 \mathrm{~nm})$ edge filter, surface temperature profiles are measured. The necessary high-dynamic range is achieved by automatic exposure control of the camera. Temperatures above $700^{\circ} \mathrm{C}$ are detectable.

2. a pyrometer measures the surface temperatures above $300^{\circ} \mathrm{C}$ at a preselectable spot of $10-\mathrm{mm}$ diameter with a time resolution of $15 \mathrm{kHz}$ (Ref. 8).

3. additionally, light can be coupled into fibers that are connected to the spectrometer outside the bunker.

\section{Experiments with Lasers}

If the upper mirror (vertical observation system) is tilted out of the straight line of sight, access is given to expose the limiter surface to laser radiation.

The LIF is applied at limiter lock 1 . The tunable dye laser pumped by an excimer laser is located outside the bunker. Mirrors guide the light to the vertical observation system. The last mirror in front of the $\mathrm{MgF}_{2}$ entrance window can be tilted to allow a scan of the laser over the whole limiter surface. The spectral range for investigations with LIF can be extended down to $115 \mathrm{~nm}$ if the powerful laser radiation produces the third harmonic in the VUV in a tripling cell filled with an Ar-Xe gas mixture and mounted onto the entrance window. ${ }^{9}$

At the upper limiter lock, a powerful Ruby laser is used for laser ablation and desorption experiments. ${ }^{10}$

For inspection, alignment, and measurements between discharges (colorimetry), in-vessel mounted halogen lamps illuminate the inserted targets.

\section{Data Acquisition}

Vacuum lock parameters such as limiter tile temperatures, reservoir pressure of the gas feed, and pyrometer signal are routinely recorded by CAMAC data loggers (Kinetic Systems) with sampling rate up to $1 \mathrm{MHz}$ inside the concrete shielding. Also, CAMAC modules provide the timing signals for triggering of the logger, gas feed, and laser pulses. The TV camera signals are recorded by microcomputer (Mikrotron) outside the bunker.

\section{SUMMARY}

The limiter lock systems at TEXTOR are user facilities that offer a wide range of applications for the investigations 
of plasma-surface interaction. Limiter and diagnostic probes can be positioned with high accuracy around the LCFS. The exchange interface for the probes provides plugs for interconnections with thermocouples, electrical power, electrical signals, and gas feed. The short time spent for target exchange gives a high flexibility for the feasibility of different experimental programs.

This is also supported by the large number of optical and spectroscopic observations from VUV to infrared obtained in vertical and tangential direction in a poloidal plane with high spatial and temporal resolution. Most of them can be measured simultaneously.

Furthermore, the coupling of measurements in both lock systems gives access to the investigation of asymmetry effects in TEXTOR. Detailed results from experiments performed at both limiter locks are given in the articles of Brezinsek et al. ${ }^{7}$ and Philipps. ${ }^{11}$

\section{REFERENCES}

1. H. SOLTWISCH et al., Plasma Phys. Control. Fusion, 36, 1A, 23 (1984).
2. U. SAMM et al., Plasma Phys. Control. Fusion, 35, 165 (1993).

3. A. COSLER, S. MUSSO, R. SCHICK, and B. SCHWEER, Proc. 18th SOFT, p. 1383, Karlsruhe, Germany (1984).

4. A. POSPIESZCZYK, Atomic and Plasma-Material Interaction Processes in Controlled Thermonuclear Fusion, p. 213, R. K. JANEV and H. W. DRAVIN, Eds., Elsevier Science Publishers B. V., New York (1993).

5. S. BREZINSEK, Jül-3962, Forschungszentrum Jülich (2002).

6. M. LEHNEN, Jül-3835, Forschungszentrum Jülich (2001).

7. S. BREZINSEK et al., "Plasma Edge Diagnostics for TEXTOR," Fusion Sci. Technol., 47, 209 (2005).

8. G. SERGIENKO, 31st European Physical Society Conf. Plasma Physics, London, United Kingdom, European Physical Society (2004).

9. PH. MERTENS and S. BREZINSEK, "Recycling of Hydrogen Isotopes in TEXTOR," Fusion Sci. Technol., 47, 161 (2005).

10. B. SCHWEER et al., "Laser Desorption of Deuterium Retained in Re-Deposited Carbon Layers at TEXTOR and JET," J. Nucl. Mater. (to be published).

11. V. PHILIPPS, "Wall Conditioning on TEXTOR," Fusion Sci. Technol., 47, 119 (2005). 